\title{
Invernadero utiliza energía solar para el control de temperatura
}

\section{Greenhouse uses solar energy for temperature control}

PÉREZ-ORTEGA, Eva Claudia†**, CHAVIRA-ÁLVAREZ, Alberto y ESPARZA-DELGADO, María del Carmen

Universidad Tecnológica de Chihuahua

ID ${ }^{\text {er }}$ Autor: Eva Claudia, Pérez-Ortega / ORC ID: 0000-0002-4739-9237, Researcher ID Thomson: S-6728-2018, CVU CONACYT ID: 388994

ID $1^{\text {er }}$ Coautor: Alberto, Chavira-Álvarez / ORC ID: 0000-0002-2705-6851, Researcher ID Thomson: S-7881-2018, CVU CONACYT ID: 250601

ID $2^{\text {do }}$ Coautor: María del Carmen, Esparza-Delgado / ORC ID: 0000-0001-8276-6031, Researcher ID Thomson: S-78232018, CVU CONACYT ID: 520791

DOI: $10.35429 /$ JTD.2019.10.3.17.23

Recibido: 10 de Marzo, 2019; Aceptado 30 de Junio, 2019

\section{Resumen}

Una empresa dedicada al ramo de hortalizas en ciudad Jiménez, Chihuahua, MX. mediante proyecto con Consejo Nacional de Ciencia y Tecnología (CONACyT) vinculado con la Universidad Tecnológica de Chihuahua desarrollan prototipo de invernadero en base al uso de energía solar con la finalidad de disminuir el uso de gas LP para el calentamiento del invernadero y la implementación de chimeneas trabajadas bajo el principio de Venturi con lo cual se estima que permite reducir el uso de ventiladores y extractores eléctricos. La construcción del invernadero es de 60 × 70 metros. El sistema electrónico del invernadero permite medir, controlar, automatizar y monitorear los parámetros de temperatura y humedad. El Control climático del invernadero es posible gracias al uso de sensores de diferentes variables climáticas como de temperatura, humedad, velocidad del viento y dióxido de carbón. La implementación del sistema solar térmico y fotovoltaico, con la sinergia de la electrónica y la automatización logra un ahorro eficiente de energía eléctrica mediante el prototipo diseñado. Objetivos: Desarrollar un prototipo de invernadero con la operación en base al uso de la energía solar para el acondicionamiento de sus instalaciones (enfriamiento y calentamiento), permitiendo una importante reducción en sus costos de operación al disminuir el uso de combustibles como gas y energía eléctrica y consecuentemente una disminución en las emisiones a la atmosfera.

Energía Solar Térmica, Sistema Fotovoltaico, Invernadero

\begin{abstract}
A company dedicated to the branch of vegetables in Jiménez city, Chihuahua, MX. Through a project with the National Council of Science and Technology (CONACyT) linked to the Technological University of Chihuahua, they develop a prototype of a greenhouse based on the use of solar energy in order to reduce the use of LP gas for heating the greenhouse and the implementation of chimneys worked under the Venturi principle, which is estimated to reduce the use of electric fans and extractors. The greenhouse construction is $60 \times 70$ meters. The electronic greenhouse system allows measuring, controlling, automating and monitoring the temperature and humidity parameters. Climate control of the greenhouse is possible thanks to the use of sensors of different climatic variables such as temperature, humidity, wind speed and carbon dioxide. The implementation of the solar thermal and photovoltaic system, with the synergy of electronics and automation achieves an efficient saving of electric energy through the designed prototype Objectives: Develop a greenhouse prototype with the operation based on the use of solar energy for the conditioning of its facilities (cooling and heating), allowing a significant reduction in its operating costs by reducing the use of fuels such as gas and electricity and consequently a decrease in atmospheric emissions
\end{abstract}

Solar Thermal Energy, Photovoltaic System, Greenhouse

Citación: PÉREZ-ORTEGA, Eva Claudia, CHAVIRA-ÁLVAREZ, Alberto y ESPARZA-DELGADO, María del Carmen. Invernadero utiliza energía solar para el control de temperatura. Revista del Desarrollo Tecnológico. 2019. 3-10: 17-23

\footnotetext{
* Correspondencia del Autor (Correo electrónico: evaperez@utch.edu.mx)

$\dagger$ Investigador contribuyendo como primer autor.
} 


\section{Introducción}

La automatización en el sistema de invernaderos inteligentes permite al ser humano la facilidad de mejorar los procesos productivos del sector primario en un ambiente protegido. La utilización de dispositivos electromecánicos, combinados con un sistema de programación y electrónica facilita que, en el proceso, aquellas variables a medir, se pueda a la vez, controlar, monitorear y automatizar.

Bajo este ambiente controlado, permite que a pesar de la sequía o de la lluvia, se tenga el proceso de producción los 365 días del año.

El utilizar ambientes cerrados y de alta tecnología, permite al productor una planeación eficaz de los alimentos, ya que dependiendo del cultivo, puede seleccionar en pantalla del equipo de cómputo, el producto a cultivar, sean tomates, chile morrón, ajo, cebolla, sólo por citar uno de los productos. En este mismo contexto se programan los cultivos con las variables que intervienen en su crecimiento y a la vez poder mantenerlas en control. Estas variables puede ser luz, humedad, dióxido de carbono, flujo de aire.

En este escenario, las pérdidas son casi nulas y cuando en zonas áridas que dependen de temporal, se pueden levantar hasta 3 cosechas en algunos productos.

Con la automatización del invernadero y en sinergia con las energías renovables, no solo se tiene un aprovechamiento por tres veces, sino que los costos implícitos de producción en el invernadero, disminuyen considerablemente por dejar de usar gas natural en la temporada de invierno, ya que para calentar se utiliza un sistema solar térmico, calefaccionando el interior por medio de agua calentada por medios solares.

La energía eléctrica utilizada por el invernadero, se provee por medio de un sistema fotovoltaico de 8000 watts interconectado a la red.

La programación del sistema hace eficiente el uso de la energía solar térmica y la energía eléctrica.

\section{Marco teórico}

La energía solar es una fuente de energía renovable, esencialmente no contaminante $\mathrm{y}$ prácticamente ilimitada.

México se localiza geográficamente entre $\operatorname{los} 14^{\circ}$ y $33^{\circ}$ de latitud septentrional, situación que resulta ideal para el aprovechamiento de la energía solar, ya que la irradiación global media diaria en el territorio nacional es de alrededor de $5.5 \mathrm{kWh} / \mathrm{m}^{2}$, colocando al país dentro de los primeros lugares en el mundo.

El clima en la región de Cd. Jiménez Chihuahua se tiene un clima extremoso lo que ocasiona que en invierno las temperaturas sean por debajo del promedio nacional, es muy bajo durante el invierno, debido a esto se tiene la necesidad de utilizar las calefacciones. El gas licuado el cual es el principal combustible que se utiliza es incosteable para la mayoría de los cultivos hortícolas y ornamentales ya que emiten gases tóxicos para las plantas.

La agricultura tradicional tiene pérdidas hasta del $40 \%$ de la producción, según las palabras del productor de chile jalapeño al que se le implementó el invernadero inteligente en la Cd. Jiménez, en Chihuahua, México.

Con las condiciones meteorológicas adecuadas, en campo abierto, con la semilla de calidad seleccionada, difícilmente se llegará al $50 \%$ de la cosecha.

Paralelo a esto, la agricultura controlada, como es el caso de un invernadero inteligente, normalmente se cosechas el $100 \%$ de las semillas.

La Agricultura Protegida es aquella que se realiza bajo condiciones en las cuales el agricultor puede controlar algunos factores del medio ambiente, esto minimiza el impacto que los cambios de clima pueden ocasionar a los cultivos.

La estructura más utilizada de la agricultura protegida son los invernaderos, malla sombra, túneles altos y bajos.

El cultivo en invernadero tiene como principales ventajas: 
- $\quad$ La protección contra el viento,

- $\quad$ Aumento de la temperatura

- $\quad$ Aumento de la superficie foliar

- $\quad$ Reducción del consumo de agua.

Marco regulatorio básico para las energías renovables:

Una política energética de largo plazo garantiza el éxito en el desarrollo de energías renovables, por lo que es indispensable contar con un marco regulatorio en materia de energía que estimule la inversión en nuevas tecnologías y garantice el desarrollo del país.

Los principales ordenamientos legales aplicables a energías renovables son los siguientes:

Constitución Política de los Estados Unidos Mexicanos.

Ley para el Aprovechamiento de Energías Renovables y el Financiamiento de la Transición Energética.

Ley de Promoción y Desarrollo de los Bioenergéticos.

Ley para el Aprovechamiento Sustentable de la Energía.

Ley Reglamentaria del Artículo 27 Constitucional en el Ramo del Petróleo.

Ley de Petróleos Mexicanos.

Ley Orgánica de la Administración Pública Federal. Ley de la Comisión Reguladora de Energía. Ley del Servicio Público de Energía Eléctrica.

Ley de Caminos, Puentes y Autotransporte Federal. Ley de Desarrollo Rural Sustentable.

- $\quad$ Ley de Energía para el Campo.

- $\quad$ Ley del Impuesto sobre la Renta.

- Ley Federal de las Entidades Paraestatales.

Ley Federal Sobre Metrología y Normalización.

Ley General de Desarrollo Forestal Sustentable.

Ley General del Equilibrio Ecológico y la Protección al Ambiente.

Reglamento de la Ley para el Aprovechamiento de las Energías Renovables y el Financiamiento de la Transición Energética.

Reglamento de la Ley del Servicio

Público de Energía Eléctrica.
Reglamento de la Ley del Servicio Público de Energía Eléctrica, en Materia de Aportaciones.

Reglamento de la Ley para el Aprovechamiento Sustentable de la Energía.

- Reglamento de la Ley de Promoción y Desarrollo de los Bioenergéticos.

- Reglamento de la Ley Federal sobre Metrología y Normalización. Reglamento de la Ley Federal de las Entidades Paraestatales.

- Reglamento Interior de la Secretaría de Energía.

A pesar de que México tiene un territorio 5.5 veces mayor que Alemania y una radiación 5.0 veces superior, la energía solar generada en el país europeo es 44.2 veces superior.

\begin{tabular}{|l|r|r|r|}
\hline \multicolumn{1}{|c|}{ País } & $\begin{array}{c}\text { Tamaño del } \\
\text { territorio } \\
\left(\mathbf{K m}^{\mathbf{2}}\right)\end{array}$ & $\begin{array}{c}\text { Radiación } \\
\text { solar } \\
\left(\mathbf{K w h} / \mathbf{m}^{\mathbf{2}}\right)\end{array}$ & $\begin{array}{c}\text { Generación } \\
\text { de energía } \\
\text { solar } \\
(\mathbf{G w h})\end{array}$ \\
\hline Alemania & $357,376.0$ & 1.1 & $5,047.0$ \\
\hline China & $9,597,000.0$ & 4.5 & $116,500.0$ \\
\hline México & $1,964,000.0$ & 5.5 & 114.2 \\
\hline
\end{tabular}

Tabla 1 Comparativo de aprovechamiento solar Notas: Por disponibilidad de información, los datos de energía solar son del 2014.

Fuentes: Elaboración por el CIEP, con datos del Bank (2017) y de SOLARGIS (2017)

Los datos del cuadro 1 considera energía solar utilite-scale es decir las llamadas granjas solares, a nivel internacional no existe una referencia que dicte a partir de cual número de metros cuadrados de paneles solares se considere utility-scale.

\begin{tabular}{|l|r|}
\hline \multicolumn{1}{|c}{ País } & \multicolumn{1}{c|}{$\begin{array}{c}\text { Penetración } \\
\text { de GD (\%) }\end{array}$} \\
\hline Alemania & 48 \\
\hline California (EUA) & 5 \\
\hline Chile & 10 \\
\hline Dinamarca & 55 \\
\hline España & 31 \\
\hline México & 0.23 \\
\hline
\end{tabular}

Tabla 2 Porcentaje de generación distribuida (GD) sobre el total de capacidad instalada

Fuentes: Elaboración por el CIEP, con datos del SENER (2017) 


\section{Metodología}

Las actividades del proyecto consistieron en el desarrollo, fabricación e instalación de un invernadero cuyo sistema de calefacción y acondicionamiento de clima se basa en colectores solares que transfieren y conservan la energía calorífica del sol a tanque termo cuyo calor a través de serpentines o intercambiadores de calor radiante será integrado al interior del invernadero. El enfriamiento del invernadero se logra a través de chimeneas de aluminio que generan una presión negativa dentro del sistema del invernadero provocando así movimiento del aire del exterior al interior el cual pasa a través de celdas evaporativas, enfriándolo y humedeciéndolo en la forma deseada y controlada. El diseño contempla desde luego un sistema de calefacción y ventiladores auxiliares eléctricos como respaldo. Para llevar a cabo el proyecto se realizaron las siguientes actividades:

Diseño de equipos: análisis histórico de temperaturas de la región, calculo y dimensionado de: estructura del invernadero, calculo y diseño de chimeneas para sistema de ventilación, calculo y diseño de sistema de colector solar para invernadero, calculo y diseño del equipo de apoyo para ambos sistemas, calculo y diseño de sistemas de conducción de agua (riego y sistema de calefacción radiante). Esta actividad se desarrolló durante los 2 primeros meses del año. Se obtuvieron planos preliminares para determinar dimensiones, características y especificaciones generales.

Ingeniería de detalle: ejecución de planos de planta y detalle de localización de: sistema de calefacción integral, sistema de ventilación integral, sistema de tubería radiante, sistema de control del invernadero. Esta actividad se desarrolló durante el tercer mes del año. Se obtuvo planos finales con dimensiones, características y especificaciones a detalle del invernadero.

Calefacción solar: Componentes del sistema de calefacción empleando energía solar:

$\begin{array}{ll}- & 8 \text { colectores solares } \\ - & 1 \text { Termotanque } \\ - & 6 \text { Intercambiadores de calor } \\ - & \text { Tubería instalada alrededor de } \\ & \text { invernadero } \\ - & 6 \text { tomas de agua }\end{array}$

Este sistema de calefacción es funcional teniendo una temperatura externa hasta de $20^{\circ} \mathrm{C}$, siempre y cuando los días se mantengan soleados y/o semisoleados.

Diseño y desarrollo de sistemas de automatización: para control y monitoreo de: temperaturas por zona, niveles de humedad, temperatura. Exterior, velocidad de interior y exterior, de colectores solares, sistema de control de aplicación de agro insumos para el desarrollo de la planta. Esta actividad se desarrolló a partir del 4to mes y estuvo mejorándose durante todo el año conforme se fue realizando las integración y prueba de los demás componentes del invernadero. Se obtuvo software y equipo para la automatización de la operación del invernadero solar el software LabVIEW fue utilizado para el desarrollo del sistema de automatización

Selección y compra de equipos, materiales de obra y consumo, elaboración de ruta crítica en base a tiempos de entrega por parte de los proveedores: esta actividad se desarrolló a partir del 4to mes y se estuvo realizando durante todo el año conforme se fue realizando la construcción e integración del proyecto. Se obtuvo el suministro de materia prima materiales, equipos y partes para la construcción del proyecto.

Fabricación de partes y elementos: esta actividad se desarrolló a partir del 4to mes y se estuvo realizando durante todo el año conforme se fue realizando la construcción e integración del proyecto (llevándose a cabo mejoras importantes al diseño inicial del proyecto). Se obtuvo la construcción de partes y elementos que conforman el invernadero y los sistemas de enfriamiento y calefacción.

Ensamble y armado de partes y elementos: esta actividad se desarrolló a partir del 4to mes y se estuvo realizando durante todo el año conforme se fue realizando la construcción e integración del proyecto. Se obtuvo el invernadero armado y ensamblado de partes y elementos

Instalación de equipo: esta actividad se desarrolló a partir del 8to mes y se estuvo realizando durante todo el año conforme se fue realizando la construcción e integración del proyecto. Se llevaron a cabo afinaciones y ajustes a los sistemas propuestos. 
Se obtuvo el invernadero con los sistemas de operación listos para la realización de las pruebas de operación.

Arranque y pruebas definitivas: las pruebas se realizaron a partir del mes 10 del año.

\section{Sistema de chimeneas:}

Componentes principales de las chimeneas:

- Lamina de aluminio y lámina galvanizada

- $\quad$ Estructura de fierro

- $\quad$ Sistema Venturi de lámina de aluminio

- Revestimiento de lana de vidrio para conservar el calor.

Capa de policarbonato que permite el paso de los rayos solares al aluminio y evita el escape del calor hacia el exterior de la chimenea.

Para obtener el caudal que fluye a través de la chimenea se tomó la lectura de la velocidad del viento y se multiplico por el área de la chimenea, en el caso de la chimenea cilíndrica, el diámetro es de $104 \mathrm{~cm}$, tomando en cuenta promedio de temperatura de 24 grados centígrados se logró un promedio de velocidad del viento de $1.5 \mathrm{~m} / \mathrm{s}$, esto dio un total de $1.125 \mathrm{~m}^{3} / \mathrm{seg}$, si se convierte esto a horas da un total de $4050 \mathrm{~m}^{3} / \mathrm{hr}$, se compara este dato con el caudal del ventilador que se utilizó para las pruebas, que tiene un caudal de $10,180 \mathrm{~m} 3 / \mathrm{hr}$. Por lo tanto, si se coloca una de las chimeneas en el lugar del extractor se debe colocar un extractor de la mitad de la capacidad del extractor del sistema celdek.

El sistema de Pared de humedad celdek incluye:

\footnotetext{
- $\quad$ Paredes húmedas

- Ventiladores

- Tuberías para dispersión de agua sobre las paredes húmedas

Bomba sumergida

Tanque de agua

Sistema de Tuberías para suministro de agua.
}

El sistema de chimeneas en conjunto con el sistema de pared húmeda y un sistema de enfriamiento a través de recirculación de agua y aire forzado logran disminuir la temperatura en un rango de 10 a 17 grados centígrados. Operando estos sistemas en forma complementaria.

El Sistema Venturi permite una corriente de aire aun mayor que la natural

\section{Sistema de colectores:}

Después analizar varias pruebas de 30 minutos de monitoreo de cada una, se observó que en promedio se logró subir $14^{\circ} \mathrm{c}$, estas pruebas fueron satisfactorias ya con el sistema de control se logra alcanzar temperaturas dentro de los rangos de $20^{\circ} \mathrm{c}$ a $25^{\circ} \mathrm{c}$ en periodos cortos de tiempo.

Las pruebas en campo demostraron que en condiciones de uso normales en la región de cd. Jiménez, el sistema podrá proporcionar hasta un 50\% de la energía necesaria para calentar el invernadero a una temperatura promedio de 22 grados centígrados dado a sus bajas tendencias de días nublados y alto contenido de radiación solar.

\section{Sistema de control:}

Los componentes que integran el sistema de automatización y control son los siguientes:

\section{- $\quad$ Unidad NI cRIO \\ - Una Gateway de red inalámbrica de sensores (wsn) NI 9795, \\ - $\quad$ tres tarjetas salida digital NI 9476, \\ - $\quad$ chasis NI cRIO 9074, \\ - $\quad$ fuente de poder NI PS-15 con un voltaje de entrada de 120VCA y voltaje de salida de 24 VDC- 5A, \\ - cable de comunicación CAT5E Ethernet, \\ - computadora PC TouchSmart-310}

Con ello se permite el desarrollo de la automatización del invernadero HombreMáquina.

Se desarrolló un sistema de adquisición de datos meteorológicos, que permite controlar y monitorear inalámbricamente las variables climáticas dentro del invernadero, con la finalidad de tener producción todo el año. 
Con el control de la temperatura, en función de los requerimientos del cultivo, se impiden excesivas temperaturas dentro del invernadero, especialmente en la primera etapa de la planta donde debe desarrollarse la aparición del primer racimo floral.

Con la automatización y control del invernadero por medio del software lobbies y hardware compact rio se logra establecer las condiciones de operación adecuadas, los cuales permiten que entren en operación los sistemas anteriormente descritos de acuerdo a las necesidades de ambientación del invernadero.

\section{Ajustes y acabados finales}

Estos se llevaron a cabo en los meses de noviembre y diciembre y permitieron realizar los ajustes necesarios a los sistemas de acondicionamiento y control del invernadero.

\section{Invernadero terminado y operando}

El invernadero estuvo terminado en el mes de diciembre y se decidió posponer la siembra del material vegetativo para el mes de marzo para la producción de plántula de pimiento (tarda aproximadamente 60 días) y posteriormente trasplantarla al sistema de producción de hidroponía (en mayo) con la intención de obtener producción a finales de julio. Obteniendo con esto dos meses más de producción que los que se obtendrían si la operación de producción se lleva a cabo en un invernadero normal.

\section{Contribución}

Obtener un prototipo de invernadero que opera a base de energía solar tanto en el calentamiento del ambiente en tiempo de frío, así como el acondicionamiento o control de temperaturas, humedades y vientos en el interior del sistema en tiempo de verano, ya que se aprovechara el bajo contenido de humedad relativa que prevalece en la región al utilizarse una pared húmeda que consta celdas Celdek de enfriamiento y chimeneas que moverán el aire del interior al exterior del sistema, por la incorporación del efecto Venturi y la radiación solar sustituyendo en gran medida el uso de energía eléctrica.
La implementación de sistemas solares, con la sinergia de la electrónica y automatización, logra un ahorro eficiente en energía eléctrica y con este prototipo, se puede mostrar a la comunidad, tanto académica, empresarial y personas en general, que el uso de sistemas solares, se pueden ir introduciendo poco a poco, con la finalidad de obtener ahorros, disminuir los costos fijos, aumentar las utilidades y poder ofrecer a la comunidad en general productos de calidad a un menor precio.

La implementación de sistemas solares, con la sinergia de la electrónica y automatización, logra un ahorro eficiente en energía eléctrica y con este prototipo, se puede mostrar a la comunidad, tanto académica, empresarial y personas en general, que el uso de sistemas solares, se pueden ir introduciendo poco a poco, con la finalidad de obtener ahorros, disminuir los costos fijos, aumentar las utilidades y poder ofrecer a la comunidad en general productos de calidad a un menor precio.

\section{Conclusiones}

En condiciones de uso normales en la región de Cd. Jiménez, el sistema podrá proporcionar hasta un $50 \%$ de la energía necesaria para calentar el invernadero a una temperatura promedio de 22 grados centígrados dado a sus bajas tendencias de días nublados y alto contenido de radiación solar.

En la práctica se detectó que la chimenea rectangular presenta un área mayor expuesta a la radiación solar y por lo tanto genera más calor que la chimenea circular. El hecho de poder generar calor con las chimeneas reduce el uso de ventiladores extractores para romper la presión de aire de la pared húmeda, dando como resultado una disminución en el consumo de energía eléctrica.

Se desarrolló por parte del equipo que participó en el proyecto CONACyT un sistema de adquisición de datos meteorológicos, que permite monitorear y controlar inalámbricamente las variables climáticas dentro del invernadero, todo ello con la finalidad de aumentar la producción deseada. 


\section{Referencias}

Agricultura protegida en México. Recuperado el 10 de abril del 2019 en: https://hidroponia.mx/agricultura-protegida-enmexico/

Alpi, A., \& Tognoni, F. (1991). Cultivo en invernadero. Mundi-Prensa Libros.

Aparicio, M. P. (2006). Energía solar fotovoltaica: guía del usuario. Marcombo.

Energía Solar en México: Potencial y aprovechamiento. Recuperado el 16 de abril del 2019

en:

https://es.scribd.com/document/350199005/Ene

rgia-solar-en-Mexico-potencial-y-

aprovechamiento\#from_embed

Iglesias Ferrer, J. M., \& Morales Salas, J. (2013). Dimensionado de un sistema térmico solar mediante simulación y su validación energética. Ingeniería Energética, 34(1), 55-65.

Hernández, J., Escobar, I., \& Castilla, N. (2001). La radiación solar en invernaderos mediterráneos. Caja Rural.

Iriarte, A., Saravia, L., \& Matias, C. (2002). Acondicionamiento térmico con energía solar de un invernadero rusticadero para la producción de plantas. In Congreso regional deficiencia y tecnología NOA. Secretaría de Ciencia y Tecnología, UNCa.

Lorenzo, E. (2002). De los archivos históricos de la energía solar Las chimeneas solares: De una propuesta española en 1903 a la Central de Manzanares. It reviews the history of the SUPPs technology.

Martinez, M. F., \& Osnaya, P. (2004). Cambio climático: una visión desde México. Instituto Nacional

Meinel, A. B., \& Meinel, M. P. (1982). Aplicaciones de la energía solar. Reverte.

Montero, J. I., \& Anton, A. (1992, October). Evolución tecnología de los invernaderos españoles. In III International Symposium on Protected Cultivation in Mild Winter Climates 357 (pp. 15-27).
Prospectiva de Energías Renovables 2012-2026. Secretaría de energía. Recuperada el 18 de abril del 2019

en: https://www.gob.mx/cms/uploads/attachment/fi le/62954/Prospectiva_de_Energ_as_Renovables _2012-2026.pdf

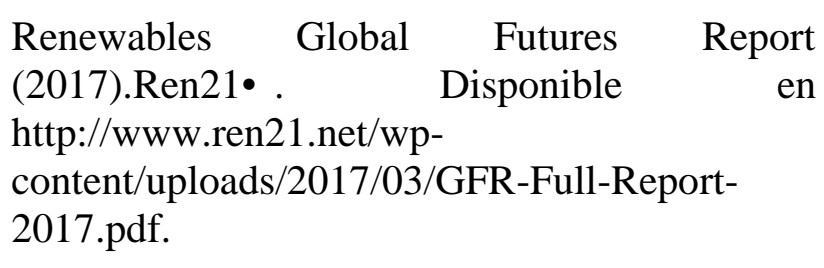

Sarmiento, P. (2007). Energía solar en arquitectura y construcción. RIL editores.

SENER (2017). Sener. Disponible en https://www.gob.mx/cms/uploads/attachment/fi le/181535/Cesar_Hernandez.pdf.

SOLARGIS (2017). Solargis. Disponible en http://solargis.com/products/maps-andgisdata/free/overview/. 